\title{
Reactive Ion Etching Process of Micro Mechanical Pendulum
}

\author{
Wei Zhang , Xiaoyan Duan, Jin Qiao and Ping Yue \\ Sensing Technology Center of Beijing Information Science and Technology University, Beijing 100101, China
}

\begin{abstract}
This paper describes the reactive ion etching (RIE) technique of micro mechanical pendulum chip. Micro mechanical pendulum chip processed by the RIE has excellent performances and surface of the chip is smoother than the chip by wet etching. Properties of chip are closely associated with the process parameters. Reactive ion etching technique improves the problems such as intricate lateral etching and surface deficiency of the wet etching. On the basis of method and many experiments of the ion etching, relation between etching rate, etching uniformity, sidewall profiles and process parameters was analyzed and a set process parameters with greater etching effect was obtained. The set process parameters includes etching depth $55 \mu \mathrm{m}$, etching uniformity (U) $0.63 \%$ and selectivity (P) $90: 1$, etching rate (V) $5.75 \mu \mathrm{m} / \mathrm{min}$, verticality of sidewall $90^{\circ} \pm 1^{\circ}$.
\end{abstract}

\section{Introduction}

Nowadays, micro mechanical system (MEMS) is an important direction of the developing-fast semiconductor industry, combining micro electronic technique with micro fabrication technique, mixing electronic and machine together. Miniaturization, high sensitivity, low cost, low energy consumption and so on are advantages of MEMS. MEMS is widely used in automobile industry, biological medicine, communications, aerospace, military and other fields [1].

Micro mechanical pendulum chip is used for sensing the inertial attitude of moving carrier and composed of silicon pendulum mass, frame and elastic torsion beam connecting silicon pendulum mass and frame [2]. Micro mechanical pendulum chip was processed by $\mathrm{KOH}$ wet etching with some disadvantages. There is a (111) surface on the silicon side forming an angle $\left(54.7^{\circ}\right)$ with (100) surface. Thus beam structure and sensitivity of micro mechanical pendulum are greatly influenced with increasing of etching depth. Lateral etching is serious in the process of wet etching and results in the low accuracy of size. In addition, reaction products of wet etching lead to more surface defects; temperature of wet etching liquor is difficult to remain stable and affects the consistency of etching rate; cost of handling waste liquid is high. Dry etching is known as another process of micro electronic technique. There are some outstanding advantages: high etching rate, high selectivity, good anisotropy, good uniformity in chip and better etching quality of profile [3]. In order to transfer patterning on mask layer to substrate with higher precision, we explore to process micro mechanical pendulum chip by reaction ion etching.
This study pays attention to analyzing relation between etching rate, uniformity, sidewall profiles and process parameters according to method and experiments, aiming to obtain ideal parameters of reaction ion etching and improve process accuracy, quality uniformity and reliability.

\section{RIE process of micro mechanical pendulum chip}

Ion etching adopts $\mathrm{ASE}^{\mathrm{TM}}$ process investigated by Bosch. Passivating and etching are conducted alternatively in the $\mathrm{ASE}^{\mathrm{TM}}$ [4]. Firstly depositing a layer of polymer keeps silicon sidewall from etching. Next polymer and $\mathrm{Si}$ are etched simultaneously. At last, keeping balance of depositing and etching realizes anisotropic etching.

Etching is carried out on the silicon substrate. Etching gas is SF6 and passivating gas is C4F8. C4F8 is passed into the chamber firstly, ionized and transformed into $\mathrm{CF}_{\mathrm{x}}^{+}, \mathrm{CF}_{\mathrm{x}}^{-}$and $\mathrm{F}^{-}$in high frequency magnetic field and induced electric field. These free radicals diffuse to surface of wafer and polymer passivation coating is formed. With SF6 introduced, there is more F- reacting with $\mathrm{Si}$ and producing $\mathrm{SiFx}$, as shown in following equations [5]:

$$
\begin{gathered}
\mathrm{C}_{4} \mathrm{~F}_{8}+\mathrm{e}^{-} \rightarrow \mathrm{CF}_{\mathrm{x}}^{-}+\mathrm{CF}_{\mathrm{x}}^{+}+\mathrm{F}^{-} \\
\mathrm{CF}_{\mathrm{x}}^{-} \rightarrow\left(\mathrm{CF}_{2}\right)_{\mathrm{n}} \\
\mathrm{SF}_{6}+\mathrm{e}^{-} \rightarrow \mathrm{S}_{\mathrm{x}} \mathrm{F}_{\mathrm{y}}^{+}+\mathrm{S}_{\mathrm{x}} \mathrm{F}_{\mathrm{y}}^{-}+\mathrm{F}^{-} \\
n \mathrm{CF}_{2}+\mathrm{F}^{-} \rightarrow \mathrm{CF}_{\mathrm{x}}^{-} \rightarrow \mathrm{CF}_{2} \uparrow \\
\mathrm{Si}+\mathrm{F}^{-} \rightarrow \mathrm{SiF}_{x} \uparrow
\end{gathered}
$$


In the experiments, same batch of double-sided polishing N100 silicon wafer (4 inches, thickness $375 \mu \mathrm{m}$ ) are used for research. Every silicon wafer is composed of $4 \times 3$ micro mechanical pendulum chip unit and lithography is performed on every unit as chrome mask shown in Fig. 1 (colored for the required etching parts). Etching opening area is less than $40 \%$ and etching depth is $55 \mu \mathrm{m}$. Conducting etching is based on DSE200E ion etching machine made by the North microelectronics company. Etching depth and shape of the window are measured by step instrument and scanning electron microscope.

Reaction chamber pressure, RF power, gas flow, ratio, etching time and passivating time are main parameters of process. These parameters determine the etching rate and uniformity. Select five points on the silicon wafer upper, middle, lower, left and right five positions as the test points and try to change process parameters and analysis the relation between these parameters and etching rate, uniformity, sidewall profile.

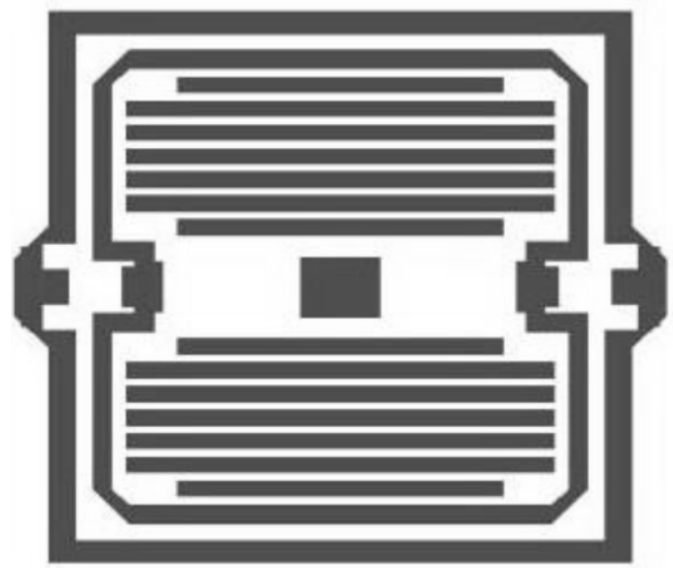

Figure 1. Chrome mask of lithography

Etching process is shown in Fig. 2. First, the silicon wafer was cleaned. Then a layer of $\mathrm{SiO}_{2}$ was formed as masking layer through oxidizing silicon wafer in the high temperature. $\mathrm{SiO}_{2}$ of every chip has same density, refractive index and other characteristics. Next, silicon wafer was etched. The process of lithography mainly includes spin-coating (speed is $2500 \mathrm{r} / \mathrm{min}$ ), prebaking $\left(90^{\circ} \mathrm{C}\right)$, exposing, developing and postbaking $\left(120^{\circ} \mathrm{C}\right)$. When etching was finished, $\mathrm{SiO}_{2}$ masking layer exposed was needed to remove. Finally, photoresist was removed and reaction ion etching was conducted on silicon.

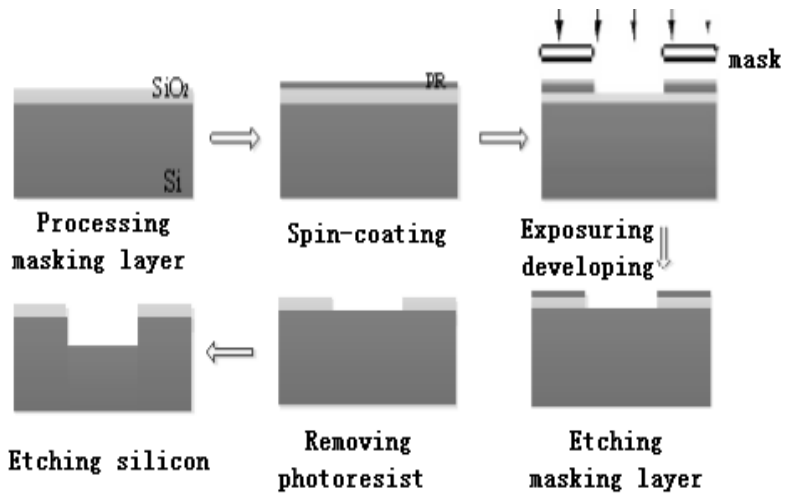

Figure 2. Etching process of micro mechanical pendulum chip

\section{Methods and results}

\subsection{Etching rate}

$$
V=\bar{D} / T
$$

$\bar{D}$ is the average value of etching depth, $\mathrm{T}$ is the total time of etching.

To study relation between ion etching rate and process parameters, etching rates were measured with different RF power shown in Fig. 3. It is presented etching rate increases with increasing of power. Increase of power results in the increase of ionization probability and more reactive particles. At the same time, there are more electrons absorbed by ungrounded RF source substrate, leading to higher negative potential, thus reaction rate is intensive and etching rate is larger. However, when the power is too high, bombarding energy will be larger and surface roughness of silicon wafer will be impacted.

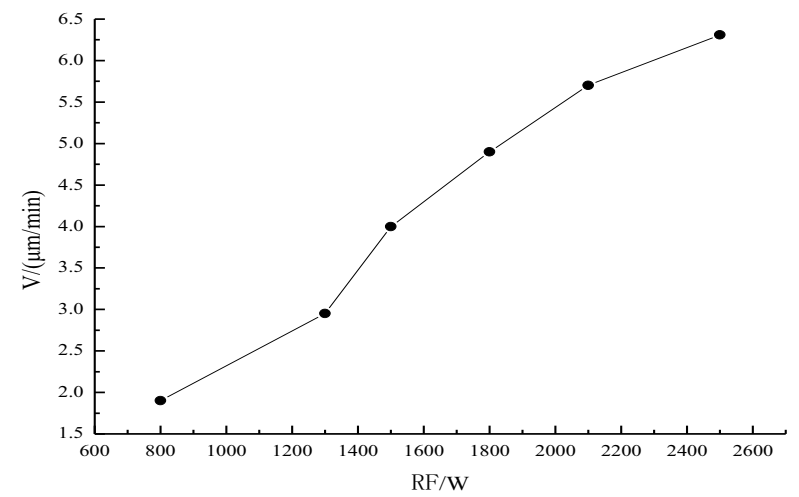

Figure 3. Relation between etching rate and RF power

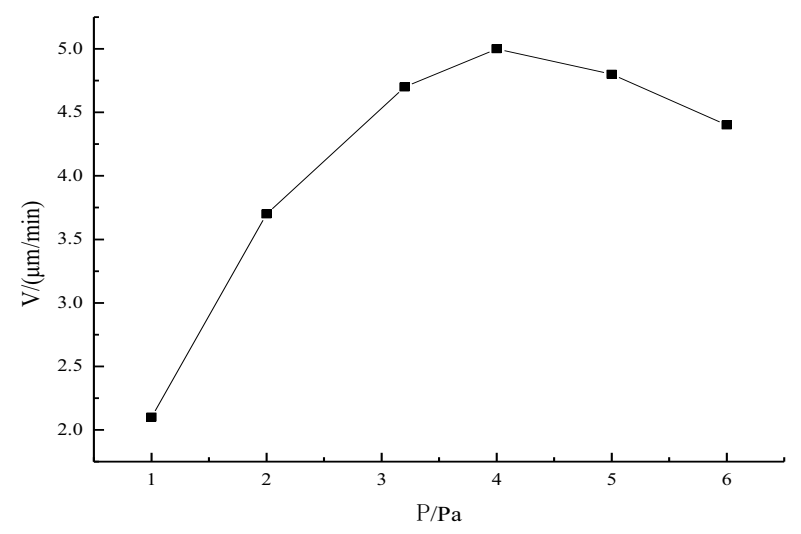

Figure 4. Relation between etching rate and chamber pressure

Etching rate is also related to pressure of process chamber, as shown in Fig. 4. If pressure is smaller, etching rate will increase with pressure rising. When pressure increases to a certain value, etching rate will dropped with pressure increasing. The reason is that increase of gas pressure results in increase of reaction gas concentration in process chamber. Consequently, chemical reaction is more intense and etching rate is quicker. With pressure increasing to a certain value, free radicals, ions and atoms participating in reaction reach saturation, and hence etching rate can not be enhanced with pressure rising. On the contrary, it shows a 
decreasing trend with energy loss caused by colliding between reaction particles. What's more, flux of reactive gas has an effect on etching rate, which is similar to the effect derived from pressure of reaction chamber.

\subsection{Etching uniformity}

Etching uniformity directly affects quality consistency of chips. Typically, etching uniformity is calculated as following equation:

$$
U=(D \max -D \min ) \div(2 \bar{D}) \times 100 \%
$$

$\mathrm{D}_{\max }$ and $\mathrm{D}_{\min }$ are the maximum and minimum of etching depth of five test points.

Silicon wafers were etched in different pressure and RF power. Etching rate of five test points are shown in Fig. 5. By calculating, it is reached that when the pressure increases, uniformity of etching will be deteriorated. Etching rate of the middle test point is slower than other test points and the difference from edge to center becomes more obvious with higher pressure. This is because the entire etching process consists of hundreds of etching cycles and etching gas is introduced in each cycle. At the moment of etching gas was passed into the chamber, test point in the middle was under the max pressure and other points were under small pressure, which caused the pressure of chamber is nonuniform. With gas in the middle flowing to around, difference of etching rate from center to edge is increasing. Therefore, etching uniformity is deteriorated.

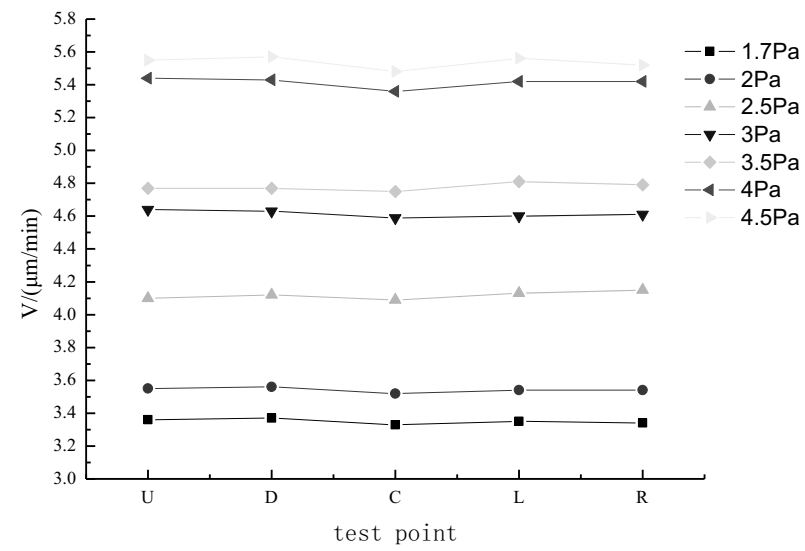

Figure 5. Etching depth of five points (U-upper, D-lower, C-middle, L-left, R-right)

\subsection{Vertically of sidewall}

Good sidewall profile is the most notable advantage compared with wet etching. Sidewall profile of chip processed in the higher electric power is shown in Fig. 6. Etching depth is larger and etching width is larger, that is to say, vertically of sidewall is over $90^{\circ}$. More reaction particles are excited as RF power increasing, but passivating gas is comparatively few and can not protect sidewall. Therefore, etching depth is larger and etching area is larger. Sidewall profile is shown in Fig. 7 with larger flow rate of $\mathrm{C}_{4} \mathrm{~F}_{8}$. Sidewall inclination is smaller and effect of etching is close to the wet etching. As flow rate of the passivating gas increases, more polymers are produced and enhance the protection of sidewall. As a result, reaction is weak and etching area is smaller with increasing of etching depth.

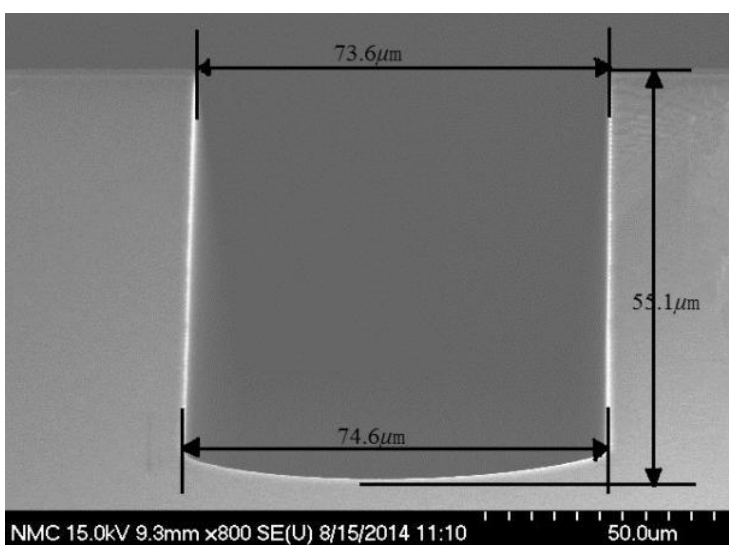

Figure 6. Sidewall profile in higher RF power

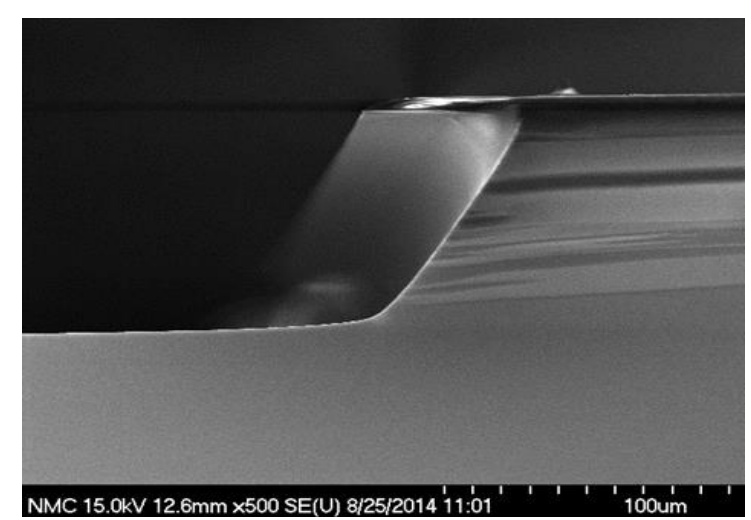

Figure 7. Sidewall profile with larger flow rate of $\mathrm{C}_{4} \mathrm{~F}_{8}$

\subsection{Analysis of ion etching}

Considering influence of parameters on etching effect and development trend, a set process parameters with good etching effect is selected in the light of experiment and shown in Table 1.

Table 1. Parameters of ion etching process

\begin{tabular}{ccc}
\hline Process parameters & Depositing & Etching \\
\hline Pressure/Pa & 4 & 4 \\
$\mathrm{C}_{4} \mathrm{~F}_{8} / 500 \mathrm{sccm}$ & 170 & 0 \\
$\mathrm{SF}_{6} / 500 \mathrm{sccm}$ & 0 & 300 \\
Loading power/W & 30 & 30 \\
$\mathrm{RF} / \mathrm{W}$ & 1600 & 2000 \\
Cycles/times & 120 & 120 \\
Time/s & 1.5 & 2.2 \\
\hline
\end{tabular}

Parameters of five points were measured by scanning electron microscope (SEM) and shown in Table 2. It can be obtained etching rate $(\mathrm{V})$ is $5.75 \mu \mathrm{m} / \mathrm{min}$ from Table 2 and Eq. (6). Uniformity (U) is $0.63 \%$, according to Eq. (7) and Table 2. 
Table 2. Measuring results of five points by SEM

\begin{tabular}{cccccc}
\hline & $\begin{array}{c}\text { Etching } \\
\text { depth } / \mu \mathrm{m}\end{array}$ & $\begin{array}{c}\text { Etching width of top } \\
\text { edge/ } \mu \mathrm{m}\end{array}$ & $\begin{array}{c}\text { Etching width of lower } \\
\text { edge } / \mu \mathrm{m}\end{array}$ & Angle ${ }^{\circ}$ & $\begin{array}{c}\mathrm{V} \\
/(\mu \mathrm{m} / \mathrm{min})\end{array}$ \\
\hline Upper & 55.0 & 75.7 & 75.9 & 89.90 & 5.75 \\
Lower & 55.1 & 75.2 & 75.1 & 89.95 & 5.76 \\
Middle & 54.6 & 75.7 & 76.0 & 89.84 & 5.70 \\
Left & 54.9 & 74.8 & 74.8 & 90.00 & 5.74 \\
Right & 55.3 & 75.2 & 75.9 & 89.64 & 5.79 \\
$\begin{array}{c}\text { Maximum } \\
\text { difference }\end{array}$ & 0.70 & 0.90 & 1.20 & & 0.36 \\
Average value & 54.98 & 75.32 & 75.54 & 89.87 & 5.75
\end{tabular}

As parameters of Table 1, the ion etching was performed and the measuring results shown in Table 2 indicate that angle of Si side section is up to $90^{\circ} \pm 1^{\circ}$ and vertically is far larger than the wet etching. Patterning on mask layer is preferably duplicated. Width of lateral etching is smaller than the wet etching, shown as Fig. 8 and Fig. 9. The black area on both sides of the damping strip is inclined plane produced by lateral etching.

Surface of micro mechanical pendulum chip is shown as Fig. 10. It is obvious that the surface is more smooth and no pits, no reaction residues comparing with the wet etching. Fig. 11 is the photo of silicon wafer surface by wet etching.

Before the ion etching, the surface of the wafer should be checked. Developing time should be accurately grasped during the etching process. The application of ion etching improves the quality of the chip, rate of finished products and reduces the problem of waste liquid treatment. However, it is needed that the equipments of ion etching are maintained regularly.
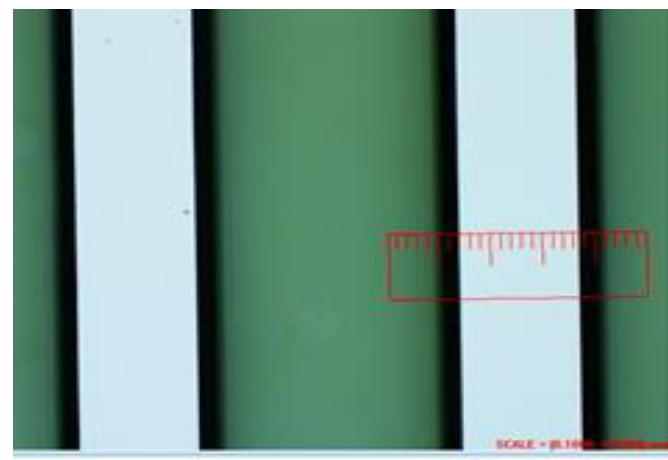

Figure 8. Damping strip of ion etching

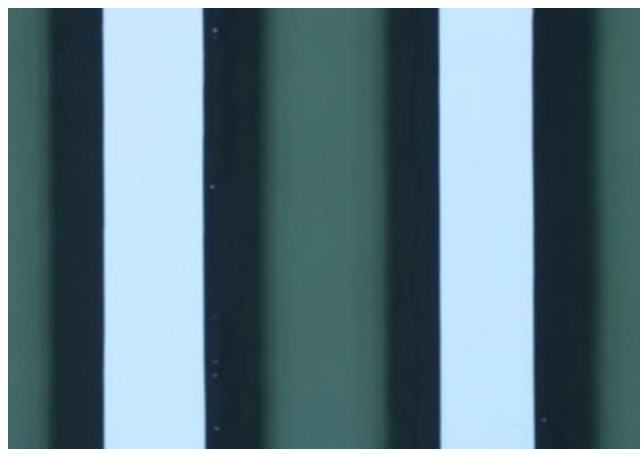

Figure 9. Damping strip of wet etching

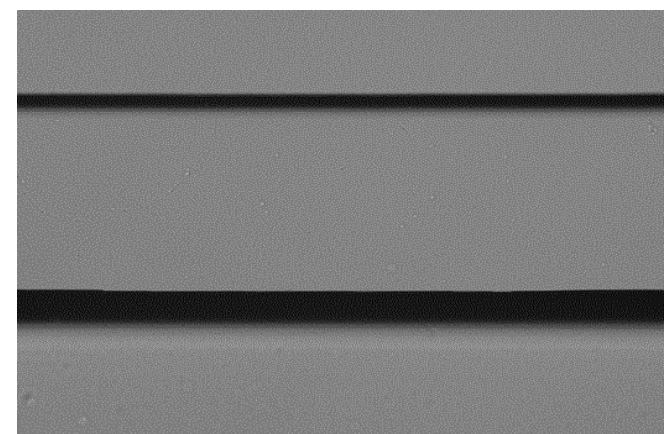

Figure 10. Surface of silicon wafer processed by ion etching

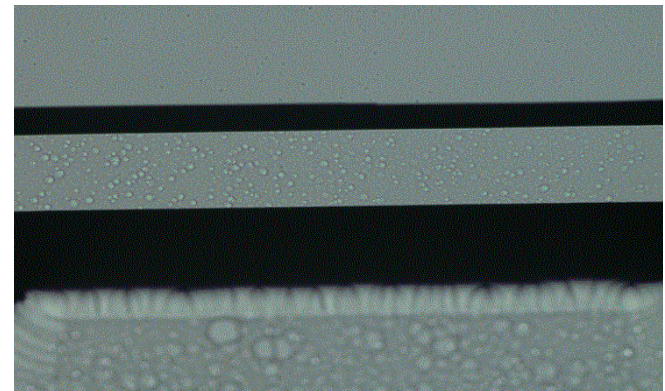

Figure 11. Surface of silicon wafer processed by wet etching

\section{Conclusion}

This study is about exploring to product micro mechanical pendulum chip by reaction ion etching. Combining experiment and method, a set parameters of etching process has been presented and it is concluded that etching rate, uniformity, sidewall profile are relevant to RF and pressure of chamber.

(1) Etching rate increases with increasing of RF and it will not increase when RF reaches a certain value.

(2) As chamber pressure is larger, etching rate increases first and then decreases.

(3) Increasing of chamber pressure causes uniformity is worse.

(4) In certain conditions, etching rate (V) is up to $5.75 \mu \mathrm{m} / \mathrm{min}$ and is about four times as large as the rate of wet etching $(1.4 \mu \mathrm{m} / \mathrm{min})[2]$, thus taping out time of micro mechanical pendulum is shorter. Vertically of sidewall $\left(90^{\circ} \pm 1^{\circ}\right)$ and uniformity $(0.63 \%)$ are good. By contrast to the wet etching, surface of micro mechanical pendulum chip is smoother and no harmful gas is 
produced during processing. In summary, reactive ion etching process provides experimental basis for improving quality consistency and reliability of the micro mechanical pendulum chip.

\section{Acknowledgment}

This research was supported by the National Nature Science Foundation of China subsidization project (61372016) and Beijing key laboratory of sensors open project fund project (2015XXX).

\section{References}

1. Z.P. Zhao. New developments in typical MEMS and wearable sensing technologies J. Micro/Nano Electronics. 1, 1-4 (2015)
2. F.X. Zhang, W. Zhang. Micro mechanical gyroscope without driving structure and its application. Beijing: National Defense Industry Press. 302-306 (2013)

3. T. Mishima et al. Crystalline anisotropic dry etching J. MEMS 2011. January 23-27. Cancun. MEXICO. 221-224 (2011)

4. X.C. Jing et al. Optimization of silica etching parameters of the dry etching $J$. Manufacturing technology. 6(1), 37-40 (2005)

5. Y. G. Li, S. J. Chen. Optimization of dry etching parameters for $\mathrm{Si}$ based on high aspect ratio $\mathrm{J}$. MEMS device and technology. 12(46), 750-754 (2009) 